\title{
LA DOCTRINA PLATÓNICA DE LOS COLORES: UNA INTERPRETACIÓN REALISTA
}

\author{
EKAI TXAPARTEGI \\ Instituto de Investigaciones Filosóficas \\ Universidad Nacional Autónoma de México \\ ekai@filosoficas.unam.mx
}

RESUMEN: Algunos de los últimos avances en el debate sobre la naturaleza de los colores han contribuido a una mejor comprensión de la doctrina platónica sobre los colores. Siguiendo esa estela revisionista, en la primera parte del artículo presento una interpretación de corte realista de dicha doctrina. En la segunda, argumento por qué ésta no puede intitularse más finamente, ni como fisicista ni como primitivista. A mi entender, ese ejercicio exegético permite justipreciar el valor contemporáneo de la doctrina de los colores de Platón, ya que las incipientes propuestas realistas pueden ahora afirmar legítimamente que el antecedente histórico que parecía faltarles se encuentra, precisamente, en los diálogos platónicos.

PALABRAS CLAVE: Platón, saturación, brillo, fisicismo, primitivismo

SUMMARY: Recent developments in the area of the metaphysics of color have led to a revision of the interpretation of Plato's doctrine. Following this revisionist trend, I present a realist interpretation of his doctrine on colors and argue why it cannot be further specified as either physicalist or primitivist. This exegetic exercise allows the appraisal of the contemporary value of Plato's doctrine on color. Incipient realist theories of color can now legitimately claim that the historical antecedent that they seem to lack can be found, precisely, in the platonic dialogues.

KEY WORDS: Plato, saturation, brightness, physicalism, primitivism

La creencia fundamental del realismo cromático, que los colores son parte del mobiliario mundano y que son ontológicamente independientes de los sujetos que los perciben, es sumamente intuitiva. Sin embargo, históricamente los filósofos modernos en su conjunto rechazaron el realismo cromático por considerarlo incompatible con la mejor descripción científica disponible de los objetos coloreados y del proceso perceptual. Es significativo que Hume, por ejemplo, tachara al realismo cromático, no sin cierta sorna, de "vulgar opinión" (Treatise, Libro I, sec. II). Sólo muy recientemente se ha introducido el realismo acerca del color en la selecta lista de ideas filosóficamente respetables. ${ }^{1}$ Eso, a su vez, ha acentuado la impresión de que es

${ }^{1}$ Se precisa la fecha fundacional de la propuesta fisicista en 1975, hace tan sólo tres décadas, cuando Smart publicó su célebre artículo "On Some Criticisms of a Physicalist Theory of Colors", el primero explícitamente fisicista. La propuesta primitivista es incluso más reciente. De hecho, todavía ni siquiera cuenta con una formulación canónica ni con un grupo de defensores claramente identificable. 
una posición que carece de tradición filosófica. Se tiende a dar por hecho que quienes afirmen que los colores son parte del mobiliario mundano y que son ontológicamente independientes de los sujetos que las perciben deben asumir su orfandad filosófica. El principal objetivo del presente artículo es mostrar que ese prejuicio histórico no corresponde con la realidad. Creo posible colegir que las teorías realistas del color también gozan de cierta tradición filosófica de la cual Platón es el máximo exponente. Con ese propósito, trataré de sacar su propuesta a la luz y argumentar que Platón concebía los colores como moradores por derecho propio del mundo natural. El segundo objetivo de este artículo es recuperar aspectos de su doctrina para la filosofía contemporánea. En este sentido, cuando menos espero poder justificar que, al igual que presentamos a Newton y a Locke como los padrinos del subjetivismo y del disposicionalismo, respectivamente, del mismo modo deberíamos comenzar a presentar a Platón como el padrino de las teorías realistas contemporáneas del color.

Antes de emprender esa tarea, sin embargo, creo conveniente recordar la razón por la cual se debería rechazar la interpretación subjetivista que tradicionalmente se ha planteado sobre la doctrina de los colores de Platón. Dicha interpretación subjetivista, que fue auspiciada por los más respetados exégetas del siglo pasado, como Taylor (1928) y Cornford (1937), está excesivamente influida por la teoría newtoniana. Ya he argumentado en otro sitio (Txapartegi 2008) que habría cierto interés filosófico no declarado en acercar la doctrina de los colores de Platón hacia la teoría subjetivista de Newton porque, entre otras razones, se asumió que la teoría newtoniana era la única concepción filosófica científicamente seria, y que cuanto más se acercara la concepción de Platón a la de Newton, más reluciría la primera. Por fortuna, esa interpretación subjetivista ya ha sido severamente contestada. McDowell (1973, p. 117), por ejemplo, objeta que no se debería atribuir a Platón el lenguaje de los sense-data. Burnyeat (1990), por su parte, cuestiona que el Teéteto se comprometa con el relativismo atributivo. Y en Txapartegi (2008) argumento, en contra de Gulley (1962), que de la analogía entre los colores y las letras del alfabeto no se sigue que Platón aceptara la tesis de la incognoscibilidad de los colores. Por eso, en este artículo no voy a ahondar en la crítica exegética. Considero que esa serie de objeciones han dejado sin su principal fundamento la interpretación subjetivista tradicional y se abre la posibilidad, que se explora en este artículo, de un Platón realista respecto de los colores. 
La situación teórica en el debate actual sobre la naturaleza de los colores ya no concibe las teorías subjetivistas como las únicas científicamente serias y eso permite una lectura de los diálogos de Platón no limitada por ese constreñimiento externo. De hecho, esa nueva situación ha beneficiado, a mi entender, tanto a la exégesis platónica como a la filosofía contemporánea. Platón debe a la filosofía contemporánea una interpretación más acorde con su concepción de los colores y menos contaminada por el subjetivismo de corte newtoniano. En la primera sección presento dicha interpretación, enfocándome sobre todo en la parte más original. A su vez, la filosofía contemporánea debe a la doctrina de los colores de Platón el hallazgo de un cotizado antecedente realista. Así, en la segunda sección argumento a favor de la legitimidad de considerar su doctrina como un antecedente realista, aunque también advierto que aplicando estrictamente la clasificación contemporánea de las teorías realistas sobre su doctrina, ésta no quedaría propiamente clasificada ni como fisicista ni como primitivista.

\section{La función del color}

A continuación presento una original interpretación de lo que Platón escribe en el Timeo (67c3-68d7) sobre los colores. Si mi interpretación es correcta, en esa parte del Timeo Platón trata de presentar el origen y la identidad más fundamental de los colores que percibimos en el mundo. Pretendía crear un modelo explicativo que, aunque hoy sabemos que es empíricamente falso, le permitiera mostrar que ciertos fenómenos cromáticos bien conocidos como la diversidad cromática, la saturación o el brillo podrían al menos tener una explicación empírica razonable. Creo que hasta la fecha esa parte sustancial de su doctrina, que nos muestra el grado de sofisticación de su concepción de los colores, ha pasado inadvertida. De ahí se seguirá, sin embargo, que su doctrina pueda ser legítimamente considerada como un antecedente interesante de las teorías realistas contemporáneas.

\subsection{De fuegos y llamas}

El principal hecho sorprendente con el que se enfrenta la explicación de la visión es la distancia espacial entre los objetos percibidos y nosotros. Para explicar cómo podemos ver desde la ladera del río las cimas de las montañas, por ejemplo, es necesario postular la existencia de algo que conecte las cimas de las montañas con nuestros ojos. Mínimamente, si no se postula ningún intermediario causal, la visión a distancia no queda explicada. O algo se traslada de allí aquí o algo se traslada de aquí allí. O, como pensaba Platón, las 
dos carreras son verdaderas. Del lado de los ojos se expande el flujo visual, que ha sido generado por la combinación entre lo que emana de nuestros ojos y la luz diurna. Esa primera fusión tiene la función de posibilitar la visión. Del otro lado, del mundo natural, provienen "ciertas emanaciones de las cosas" (Menón 76c6-7), i.e., los fuegos o las luces que irradian los objetos constantemente. Así, cuando el flujo visual, convertido ahora en un cuerpo que temporalmente se extiende del órgano visual, intercepta esos fuegos que provienen de los objetos surge la visión (Timeo 45b2-d3), ${ }^{2}$ que es característicamente cromática.

Creo entender que ambos tipos de flujos (el flujo visual y el flujo objeto) se insertan en la teoría de la visión para explicar la distancia entre lo percibido y el sujeto que percibe visualmente. Es interesante ver, sin embargo, que al flujo objeto también se le asigna otra función, i.e., explicar la naturaleza del color. ${ }^{3}$ Es decir, según el Menón (76d4-5), los colores son (o, al menos, se encuentran entre) aquello que procede constantemente de los objetos: "El color es una emanación de las figuras, proporcionado a la vista y, por tanto, perceptible." En el Timeo (67c6-7), Platón repite básicamente la misma afirmación. El color es la "llama que fluye de cada uno de los cuerpos y con sus partículas proporcionales a nuestra visión posibilita la percepción". Es natural leer esos pasajes como definiciones nominales que nos indican cuál es la naturaleza real de los colores. El color es idéntico al flujo de partículas (o figuras) que sale del objeto (Taylor 1928, pp. 277-278). En la visión, la función explicativa de la llama que sale del objeto sería la misma que la función epistémica del color, i.e., posibilitar la percepción visual de los objetos distantes así como la de sus propiedades. ${ }^{4}$ La explicación natural de la atribución cromática es, por lo tanto, bastante sencilla: vemos los objetos durante el día y vemos cómo son porque hay un flujo, una llama o una emanación de partículas (o figuras); es decir, el color, que se traslada constantemente desde cada uno de ellos. Al igual que la luz diurna es la condición de posibilidad para que podamos ver los colores, porque sin él no habría flujo visual, los colores son la condición de posibili-

\footnotetext{
${ }^{2}$ Platón relaciona esta idea con Empédocles (Menón 76c6-7), aunque hay matices relevantes entre ambos filósofos (véase Ierodiakonou 2005, pp. 220s, o también Taylor 1928, p. 480).

${ }^{3}$ Al flujo visual también se le asigna otro papel: explicar por qué creemos percibir algo mientras dormimos (Timeo 45d5-46a2).

${ }^{4}$ Véase también Platón, Carmides 168d9-10. Aristóteles critica esa idea en De Sensu (437a-b). En el De Coloribus (79 ${ }^{I}$ bl5-17), en cambio, quizá el propio Aristóteles se defiende.
} 
dad para que podamos ver los objetos y sus propiedades a distancia, porque el flujo visual únicamente detecta colores. ${ }^{5}$ La función del color es posibilitar la visión a distancia.

\subsection{Intuiciones prenewtonianas}

Con lo expuesto hasta ahora se hace evidente la falta de paralelismo entre la propuesta platónica y la newtoniana, con la cual normalmente operamos. Según Newton, prácticamente toda la luz natural es solar, proviene de la misma fuente, el Sol, y los objetos no hacen sino absorber/rebotar partes de esa luz. Según Platón, sin embargo, hay varios tipos de luces (en última instancia, varios tipos de fuegos), cada una con su propia fuente: la diurna, la de los objetos y la del ojo. ${ }^{6}$ Por lo tanto, la luz que irradian los objetos, el color, no es la luz diurna reflejada. ${ }^{7}$ Resaltar esta falta de paralelismo es importante en un doble sentido. Por un lado, se hace palpable la distancia conceptual entre la concepción de los colores de Platón y nuestro "sentido común científicamente ilustrado" respecto de los colores. Esa distancia explica, a mi entender, por qué se nos hace tan difícil imaginarnos con exactitud cómo concebía Platón los colores. Pero, por otro lado, nos muestra que a pesar de que entendía de una manera diferente de la nuestra conceptos-marco como "luz", "ojo", "color", etc., su propuesta era altamente intuitiva y, como veremos, era capaz de proponer soluciones económicas y sencillas a los principales problemas empíricos que rodean la naturaleza de los colores.

${ }^{5}$ Por eso, el hecho de que la luz irradiada por los objetos (un fuego no puro y fiero) sólo se pueda percibir cuando la atmósfera está llena de luz diurna no implica que, para existir, la primera dependa de la segunda. La luz diurna sólo es constitutiva de las condiciones que posibilitan la percepción de los objetos (porque constituye el flujo visual). La dependencia de la luz que irradian los objetos respecto de la luz diurna es epistémica (supone una condición necesaria para que puedan ser vistos), no ontológica (la luz que sale de los objetos no es ni se constituye de luz diurna). Véase también Cornford 1937, pp. 152-153.

${ }^{6}$ El contraste entre los dos tipos de fuegos lo hace en el Timeo 58c5-dl, la luz del día sería un fuego puro, no así el fuego que emanan los objetos. Por otra parte, la luz del día no emana de los objetos, sino de las llamas, haciéndolas visibles. Véase también Cornford 1937, p. 152. Otra consecuencia de esto es que todos los objetos visibles estarían compuestos por el tipo de fuego que no quema, incluso el agua o la tierra, porque de lo contrario serían invisibles (Timeo 3lb5).

${ }^{7}$ Para apreciar esa importante diferencia entre los dos paradigmas, conviene recordar que, contrariamente a lo que sabemos, en Platón la metáfora de la irradiación se aplica más naturalmente a las luces de los objetos y a las del ojo que a la luz diurna que, como la noche, se imagina como un cuerpo en perpetuo movimiento cíclico (Timeo 39b2-c2). 
Gracias a la ciencia moderna, hoy sabemos que la teoría de la percepción que Platón manejaba es empíricamente falsa. El flujo visual no existe, por ejemplo. El flujo objeto no tiene como fuente suficiente el propio objeto, etc. No obstante, hay al menos dos razones de principio por las cuales la identificación del color con la llama del objeto parece apelar positivamente a nuestras intuiciones. La primera razón que la hace atractiva es su consistencia con la fenomenología del fuego real. El fuego, a diferencia de la tierra, el agua o el aire, es un elemento que se siente a distancia. Cuanto mayor es el fuego, mayor es el calor que despide y mayor es la distancia en la que se siente. Por lo tanto, un objeto que se está quemando se siente desde más lejos que uno que no se quema. La función explicativa del fuego (que no quema) para la visión sería, por lo tanto, detectar incluso a distancias muy lejanas los objetos que constantemente despiden dichos fuegos. En ese sentido, no cabe duda de que la hipótesis de que los objetos están constituidos por fuego que se expande constantemente de ellos es ingeniosa al permitir explicar la percepción visual de los objetos distantes de una manera sencilla e intuitiva.

Segunda, en comparación con la explicación newtoniana, esa explicación tiene una mayor consistencia con el uso atributivo habitual de los conceptos cromáticos. En particular, no tiene que recurrir al disposicionalismo para dar cuenta del color de los objetos. La razón es que, para Newton, el hacedor-de-color es la luz reflejada que no pertenece al objeto, mientras que para Platón el color es la llama que es parte del objeto. ${ }^{8}$ Así, la llama de fuego no es un ente separable del objeto que se quema. Objeto y llama pueden concebirse como una unidad porque el fuego se atribuye a los objetos sensibles que la contienen, mientras que, en el modelo newtoniano, el objeto es un ente y la luz reflejada es otro ente ontológicamente distinguible. ${ }^{9}$ En consecuencia, el modelo griego engarza de manera más natural que el newtoniano con el uso atributivo habitual de los conceptos cromáticos (así como de los sonidos y los olores). Los colores se identifican con las emanaciones de los objetos que tienen como función hacer visible todo el objeto ${ }^{10} \mathrm{y}$, evidentemente, eso implica que es legítimo juzgar

\footnotetext{
${ }^{8}$ Struycken (2003, p. 297) también interpreta los flujos que salen de los objetos como sus "partes".

${ }^{9}$ El fuego, normalmente, se identifica con la llama pero la llama no se quema, se quema el objeto. Siempre que hay fuego, hay algún objeto que se está quemando. Hablamos así. Atribuimos el fuego al objeto, no a la llama.

${ }^{10}$ La función cognitiva del color es pasiva: que el objeto pueda ser visto a distancia. La función del ojo, sin embargo, es activa en el sentido de que busca la interacción con los objetos, detecta su color y transmite esa información (a través
} 
el color como parte de esos objetos visibles. A diferencia del modelo newtoniano, el modelo griego no necesita comprometerse con una reelaboración semántica disposicional que explique la legitimidad de la atribución de los colores a los objetos. Si los colores son llamas o eflujos, entonces son categóricamente atribuibles a los objetos que las despiden a pesar de que se extienden más allá de los límites corporales de éstos.

\subsection{En blanco y negro (Timeo 67d2-e6)}

A continuación analizaremos la correlación que Platón establece entre la diversidad cromática y los diferentes tipos de emanaciones de los objetos. Respetando el orden que nos propone, comenzaremos por el blanco y el negro:

Las partículas que proceden de los otros cuerpos y afectan la visión son, unas, menores, otras, mayores y otras, iguales a las partículas visuales propiamente dichas. Las iguales son imperceptibles, las que denominamos transparentes; en cuanto a las mayores y las menores, aquéllas contraen el rayo visual, éstas lo dilatan, similares a los calores y fríos en la carne, a las sustancias astringentes en la lengua y a todo lo que llamamos punzante por producir calor; lo blanco y negro, aunque son los mismos fenómenos que aquéllos, parecen diferentes por darse en otro nivel. Hay que designarlos como sigue: lo que tiene la propiedad de dilatar el rayo visual es blanco; negro, su contrario. (Timeo 67d2-e6)

Según esta cita, hay dos tipos de flujos no transparentes que provienen de los objetos, el ancho y el estrecho. ${ }^{11} \mathrm{El}$ ancho se identifica con el negro y el estrecho con el blanco. La explicación del blanco y del negro, por lo tanto, es aparentemente sencilla. Los objetos negros son los que despiden las partículas más grandes, y los blancos, al contrario, los que despiden las partículas más pequeñas. Sin embargo, esta explicación aparentemente tan sencilla genera un gran problema: impide la explicación de la riqueza cromática. Si se identifican los flujos de los objetos con los colores, y éstos lógicamente no pueden ser sino más anchos o más estrechos que el flujo visual, entonces sólo puede haber dos colores, el blanco ( $\lambda \varepsilon \cup \varkappa o \nu)$ y el negro $(\mu \varepsilon \lambda \alpha \nu)$. Es decir, si entendemos "blanco" y "negro" a nuestro modo occidental,

de todo el cuerpo) al alma. No obstante, no es tan activa como en el modelo de Newton donde el ojo (o la mente) "genera" o "constituye" los colores de los objetos por medio de las sensaciones subjetivas.

${ }^{11}$ Véase también Menón 76dl. 
como tonos cromáticos, entonces la caracterización del blanco y del negro que se hace en ese pasaje es evidentemente falsa, porque su resultado sería un mundo en blanco y negro, y nuestra visión, típicamente, no es monocromática. Es decir, si los colores son flujos y sólo hay dos tipos de flujos no transparentes, ya que éstos se definen en comparación al grosor del flujo visual, entonces sólo puede haber dos colores, porque todos los tipos de flujos se pueden clasificar, en última instancia, como "o más anchos o más estrechos" que el flujo visual y, en consecuencia, serían o blancos o negros. Identificar los tonos blanco y negro con las anchuras relativas de las partículas de los flujos conduce la doctrina de Platón a un absurdo demasiado evidente, incompatible con decir que el cielo es azul.

Una vía de solución que se ha explorado, también de dudosa consistencia, ha sido suponer que Platón explica la diversidad de los colores en el mundo por "las diferencias que resultan del encuentro" entre los dos flujos, el visual y el que proviene de los objetos (Taylor 1928, p. 480). Sin embargo, según esa cita el resultado de ese encuentro también se limita a dos efectos, estrechar o dilatar el flujo visual, y, por lo tanto, a esos dos mismos colores. No parece que podamos avanzar mucho en esa dirección. Por eso, quizá, hay quien ha leído esa parte del Timeo en relación con una doctrina muy generalizada en su época, según la cual sólo hay dos colores primarios, el blanco y el negro. Todos los demás colores serían derivados, fruto de las mezclas de diferentes proporciones entre estos dos colores (Struycken 2003, p. 279). No obstante, creo que ni siquiera así llegamos al resultado deseado. El problema de cómo extraer la variedad cromática persiste, porque si la identificación nominal es verdadera, el blanco y el negro no sólo serían los dos colores primarios; serían también los dos únicos colores.

Los defensores de esta segunda estrategia podrían todavía insistir en su interpretación y rechazar que los colores secundarios se identifiquen con los flujos. Sin embargo, eso nos conduciría a otro problema incluso mayor porque, sensu stricto, los colores secundarios dejarían de ser colores - porque ya no serían emanaciones de figuras - . O, a la inversa, seguir considerándolos colores —a pesar de no ser emanaciones - implicaría elaborar una concepción más compleja de la naturaleza de los colores. La naturaleza de los colores ya no sería unitaria, habría varias naturalezas dependiendo de cuál sea el color. Algunas serían emanaciones; otras no. Y eso origina un dilema. Mientras se abre la necesidad de complementar la teoría para poder explicar los colores que no son emanaciones (todos excepto el 
blanco y el negro), las ventajas explicativas y semánticas que obtendríamos identificando los colores con los flujos quedarían limitadas a dos colores, el blanco y el negro. En definitiva, el problema sería que no sabemos con qué se podrían identificar los demás colores para que puedan ser vistos a distancia y, además, ser legítimamente atribuibles a los objetos.

Afortunadamente, hay al menos otra interpretación que no se debería descartar. El blanco y el negro no son tonos cromáticos sino que representan los límites de la escala de saturación en la que se encuentran los demás colores. Es decir, al describir dos tipos de partículas, Platón no estaría identificando dos tonos cromáticos sino que, al igual que Aristóteles, estaría ofreciendo la escala en la cual integrar los demás colores. ${ }^{12}$ Del mismo modo en que nosotros podemos clasificar los colores como más "claros" o más "oscuros", los griegos podrían clasificar los demás colores como más "blancos" o más "negros" dependiendo de si sus partículas son más anchas o más estrechas que las del flujo visual (Gage 1993, p. 11). Esto no excluye el blanco y el negro como colores tonales. Podrían quedar caracterizados, por ejemplo, como colores terminales de una escala de saturación, el negro sería el color más oscuro y el blanco el más claro.

1.4. Una explicación para la saturación, otra para el brillo y otra para. . . los demás

Generalizando la propuesta que acabo de realizar, creo que a la hora de interpretar correctamente el pasaje relevante del Timeo (67c368d7) tienen especial importancia tanto el orden en el que va colocando los colores como el tipo de explicación que les va asignando. En particular, creo que los trece colores que menciona se pueden clasificar en tres bloques dependiendo de sus respectivas explicaciones. El primer bloque lo componen el blanco y el negro (los cuales, a mi entender, equivalen a la saturación o a la claridad/oscuridad de los colores) que se explican, como acabo de señalar, por el tamaño

12 Taylor, van Brakel y Saunders también establecen una línea de continuidad entre esta sugerencia platónica y la escala cromática aristotélica: "Timeo trata los colores en una escala linear donde el blanco y el negro son los dos colores terminales, al igual que Aristóteles y Goethe lo harán tras él" (Taylor 1928, p. 481); "Aristóteles usó una escala de siete grados: blanco, amarillo, verde, azul oscuro, púrpura, rojo y negro. La mayoría de las escalas desde Aristóteles hasta Grosseteste (siglo XIII) contienen siete colores que van del blanco al negro en una escala linear, aunque en cada caso varían los términos y el orden" (van Brakel y Saunders 2002, p. 319; las traducciones son mías). 
(o grosor) relativo de sus partículas. El segundo bloque, de un solo miembro, lo compone el brillo que se explica por la velocidad relativa de las partículas:

El movimiento más agudo, perteneciente a otro género de fuego, que dilata el rayo visual hasta los ojos, abre con violencia sus salidas y las funde en una masa de fuego y agua, que llamamos lágrima cuando desde allí se vierte. La misma es fuego y se encuentra con fuego que avanza desde el lado contrario. Cuando un fuego salta como un rayo mientras otro entra y se apaga en la humedad y, en esta conmoción, nacen múltiples colores, llamamos a este fenómeno destellos y denominamos a lo que lo produjo brillante y esplendoroso. (Timeo 67e6-68bl)

Parece seguro afirmar que si "el movimiento más agudo" dilata el rayo visual y abre con violencia sus salidas para producir la sensación de brillo entonces las partículas que se desplazan lentamente no dilatan el rayo visual y, por lo tanto, no pueden ser brillantes. Una condición necesaria (y seguramente suficiente) para que algo sea visto como brillante es que las partículas que componen su emanación sean partículas que se trasladen rápidamente. Al menos tan rápidamente como para dilatar el flujo visual. En consecuencia, el torrente de partículas más dinámico es el que se ve más brillante.

Más adelante veremos en detalle el tercer bloque, compuesto por diez colores particulares ${ }^{13}$ que se explican, aparentemente, por la combinación de otros colores. Antes sin embargo, conviene resaltar que hay una clara diferencia explicativa entre los tres primeros colores (blanco, negro y brillo) y los siguientes diez que no parece haber sido suficientemente apreciada. ${ }^{14}$ Los tres primeros no se explican mediante la combinación de otros colores, como hará con los diez

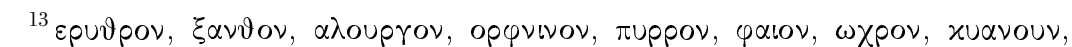
$\gamma \lambda \alpha u x o \nu, \pi p \alpha \sigma \iota v o \nu$. Varía según la traducción (basta comparar dos, la de Lisi y la de Samaranch — entre paréntesis-), pero normalmente en español se presentan como rojo, castaño rojizo (o amarillo oro), púrpura, gris amarronado (o pardo oscuro), rojo amarillento (o pardo claro), gris, amarillento (u ocre claro), azul oscuro, verde azulado y verde suave (o verde oliva). Por desgracia, a veces parece que la traducción castellana está influida por un intento de "dar sentido" a los resultados de Platón, desde la suposición de que su pretensión era describir las mezclas de pigmentos.

${ }^{14}$ Taylor, por ejemplo, considera que el tamaño y la velocidad relativa explicarían la variedad cromática, cuando en realidad el tamaño sólo lo menciona en relación con el blanco y el negro, y la velocidad sólo en relación con el brillo y el rojo-sangre. "Platón también da cuenta de la variedad de los colores que vemos, admitiendo partículas de fuego de diferente tamaño así como diferentes velocidades de propagación" (Taylor 1928, p. 487; la traducción es mía). Además, Taylor incurre en una 
restantes, sino mediante el tamaño y la velocidad relativa de las partículas. Y aquí es posible trazar un evidente paralelismo que, quizá, ha pasado inadvertido. El tamaño relativo, al igual que la velocidad relativa, son características que, en principio, se pueden aplicar a cualquier tipo de partículas (o figuras). Empíricamente, todos los tipos de partículas pueden ser más o menos anchos o más o menos rápidos que las partículas del flujo visual. Es decir, los primeros tres "colores" que se tratan en el Timeo, no parecen ser "colores tonales" propiamente dichos, sino cualidades de los colores que se identificarían con lo que nosotros denominamos saturación y brillo. Es por eso que serían caracterizados como aplicables a cualquier flujo. Como todos los flujos están compuestos de partículas que son más o menos anchas y más o menos veloces que las del flujo visual, todos los colores particulares serán más o menos saturados y más o menos brillantes.

Si esto es así, no sería correcto suponer, por ejemplo, que hay dos (o tres) colores primarios de los cuales se extraen todos los demás colores (Cornford 1937, p. 277; Ierodiakonou inédito). El blanco y el negro serían cualidades de los colores, no colores tonales en el sentido en que nosotros los entendemos. Tampoco sería correcto suponer que, para Platón, el "brillo" era un color tonal. ${ }^{15} \mathrm{Si}$ la hipótesis es verdadera, el brillo, junto con la saturación, se podría aplicar a los demás colores particulares. Así, se desharía la sorprendente interpretación que dice que Platón tenía un nombre para "brillo" pero que lo consideraba un color tonal, cuando es un hecho evidente, suponemos que también para los griegos, que el tono "brillo" no existe. Al contrario, es una propiedad que se puede asociar con muchos colores. ${ }^{16}$ No sería justo atribuir a Platón la afirmación de que el color tonal "brillo" existe cuando aquello que brilla o hace brillar es, precisamente, el color.

contradicción con su posición original, ya que, según esto, la riqueza cromática no se explica por cómo impactan dos tipos de flujos cromáticos en el flujo visual, sino por cómo modifican el flujo visual toda una variedad de flujos cromáticos que se distinguen entre ellos por su forma y velocidad.

15 "Sin embargo, lo que trato de subrayar aquí es que, en contra de las intuiciones modernas, Platón obviamente piensa que no solamente el rojo sino que también el brillante es un color" (Ierodiakonou inédito; la traducción es mía).

${ }^{16} \mathrm{El}$ brillante se asocia a toda clase de colores, como queda claro en la propia cita. Pero hay más ejemplos. Platón asocia el brillante con el amarillo cuando dice que el oro es brillante y amarillo (Timeo 59b4-5). Si Platón entendía el brillo como un color tonal, el oro tendría dos tonos y, por lo tanto, no estaría describiendo la superficie homogénea del oro. El resultado de aplicar el brillo como color tonal a ese pasaje sería, al menos, sorprendente. 


\subsection{La materialidad del color}

Hemos llegado al resultado de que Platón también consideró (y trató de explicar) al menos dos dimensiones o cualidades de los colores, como son su saturación y brillo. Y lo hizo además de una forma maestra, separando los dos tipos de explicaciones. La saturación y el brillo los explica, al parecer, por las características relativas de las partículas respecto del flujo visual, por su tamaño y velocidad relativas. Ahora nos queda por ver cómo explica Platón la naturaleza de cada flujo o color particular. Para ello me apoyo en la propuesta de Struycken (2003), según la cual los colores particulares griegos se asocian a su soporte material de una manera esencial. La idea es que los diez colores particulares no se generan combinando pigmentos o colores primarios, como se ha creído tradicionalmente (Cornford 1937, pp. 276-279; Taylor 1999, p. 117), sino que se generan a partir de las posibles combinaciones entre los cuatro elementos básicos que conforman los objetos naturales (fuego, agua, tierra y aire) y sus posibles transformaciones ${ }^{17}$ (ya que Platón no toma los cuatro elementos como fundamentales y permite que se transformen unos en otros). ${ }^{18}$ La combinación de estos elementos básicos genera una multitud de objetos naturales cada uno de los cuales posee una apariencia cromática característica en el acto de ver. Los objetos naturales, por lo tanto, están constituidos por ciertas partículas elementales las cuales dependiendo seguramente de su forma, ${ }^{19}$ composición y proporción determinarán el aspecto cromático del objeto que las despide. De ahí se sigue que para saber cómo se genera un determinado color, debe preguntarse primero cómo se genera un determinado objeto. Y así es precisamente como Struycken lee el pasaje correspondiente a los colores del Timeo, como una descripción de los procesos naturales que generan los objetos que serán característicamente "azules", "amarillos", etc. (y no como una descripción de las mezclas entre pigmentos azules, amarillos, etc.). Concretamente, el primer ejemplo

${ }^{17}$ Struycken (2003, p. 277) recupera esa idea del pasaje del De Coloribus: "Éstos [se refiere a tres elementos: fuego, aire y agua] mezclados en mayor o menor proporción, producen por así decirlo todos los colores" ([Pseudo-Aris.], De Coloribus, $792 b$; la traducción es mía).

${ }^{18}$ Los elementos son fijaciones temporales del "receptáculo" (Timeo 5la4-6).

${ }^{19}$ Platón afirma que lo que entra al flujo visual debe ser un fuego puro, porque el fuego interior es puro y sólo lo semejante puede afectar a lo semejante (Timeo 45b6d3). Por lo tanto, parece que la única forma cromática detectable por el flujo visual es la pirámide (Ierodiakonou 2005, pp. 223-224; pace Struycken 2003, p. 277), a pesar de que puede haber distintos tipos de pirámides dependiendo de sus ángulos, de su tamaño o de si están enteras. 


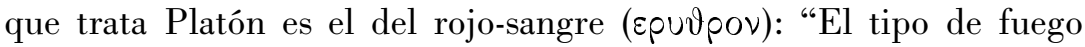
intermedio es el que, a pesar de mezclarse con la parte húmeda de los ojos, cuando la alcanza no es resplandeciente. Aplicamos el nombre de rojo al rayo de fuego mixto que atraviesa la humedad y da un color sangre" (Timeo 68bl-5).

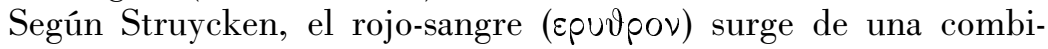
nación de las partículas de fuego y humedad. La sangre tendría el aspecto rojo porque es un fuego-mojado o un líquido fogoso que contiene la combinación de ambas partículas (las pirámides del fuego y los icosaedros del agua). Según esto, el flujo exterior está compuesto únicamente de pirámides (Ierodiakonou 2005, pp. 223-224) y los objetos que las emanan se ven rojos cuando el ojo alcanza a añadir los icosaedros. Esta hipótesis depende de que las figuras de las emanaciones sean solamente pirámides, hipótesis que no podemos confirmar. Sin embargo, si fuera cierta, podría ser el caso de que no hubiera objetos rojos en realidad y que cualquier objeto que se viera rojo tuviera un componente subjetivo inherente (los icosaedros que la lágrima añade). ¿Implica eso que todos los casos de percepción de rojo son ilusorios? No necesariamente. Imaginemos el siguiente escenario. La sangre está compuesta de pirámides de diverso tamaño e icosaedros. Por eso es roja. Ahora bien, los icosaedros no son parte del flujo que despide la sangre (porque éste, según la hipótesis, sólo se compone de pirámides). En ese caso, la intervención de la lágrima no viene sino a recomponer su composición original, añadiendo a las pirámides aventureras los icosaedros que no se atrevieron a navegar por el espacio. La función del ojo, así como la función de las pirámides que provocan la intervención de la lágrima, sería precisamente optimizar la percepción de los colores en el mundo. ${ }^{20}$

El segundo color particular sería, según Struycken, el color del sol,

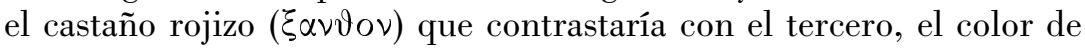
la noche, el púrpura ( $\alpha \lambda$ ouprov): "El brillante mezclado con el rojo y el blanco es castaño rojizo. [...] Ciertamente, el rojo, mezclado con el negro y el blanco produce el púrpura" (Timeo 68b5-6 y 68b8-c1).

Si la hipótesis anterior es cierta, el castaño rojizo se compondría de icosaedros y pirámides grandes que se trasladaron a gran velocidad. El púrpura también contendría pirámides e icosaedros, sólo que en este caso algunas pirámides serían grandes y otras pequeñas y todas

\footnotetext{
${ }^{20}$ Una consecuencia no deseable de esta lectura es que si los elementos de la tierra y el aire no se encuentran dentro del ojo y, por lo tanto, no intervienen, parece que cualquier objeto que las contenga generará una ilusión cromática, pues en esos casos no se podrá compensar el hecho de que sus respectivos flujos se componen únicamente de pirámides.
} 
ellas se desplazarían lentamente. A continuación, Platón afirma que cuando éstos se queman y se les añade más negro, se crea la cuarta combinación cuyo resultado es el gris amarronado (opழvivov). A partir de aquí los procesos naturales se complican, y con éstos la descripción exacta de las correspondientes composiciones. Así, el siguiente color es el rojo amarillento (tuppov) que la luz del Sol (el castaño rojizo) adquiere, según la interpretación de Struycken, cuando pasa por algún tipo de filtro o humo (gris). El sexto, el gris (yaı) ) se obtiene mezclando el negro y el blanco como, por ejemplo, en las transiciones del día y la noche. En la séptima mezcla, entre el castaño rojizo y el blanco, según Platón, se obtiene el amarillento u

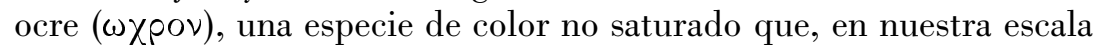
cromática, podría abarcar del rojo al naranja o, incluso, a algún tipo de verde (Gage 1993, p. 12). Aquí es donde se manifiesta con mayor claridad que Platón no está mezclando tintes sino describiendo algún proceso natural, como quizá la pigmentación de la piel humana que empieza siendo blanca pero que bajo la luz del Sol va tornándose de un color rosado no saturado. ${ }^{21}$ El siguiente color que Platón trata

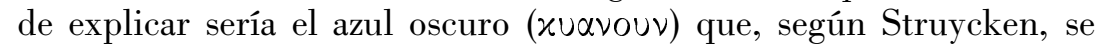
genera por el desplazamiento del color oscuro de la noche al mezclarse con la brillante luz blanca del día. Las dos últimas génesis cromáticas que Platón trató de describir se encontrarían, según Struycken, en el arco iris. Según el quinto proceso, el blanco oscurecido por algún filtro gris, se torna rojo amarillento. Si éste continúa oscureciéndose, como en la figura del arco iris, da un verde azulado ( $\gamma \lambda \alpha u x o \nu)$ que, a su vez, continúa gradualmente oscureciéndose y acercándose a un verde suave ( $\pi \rho \alpha \sigma i v o \nu)$.

En resumen, lo que podría explicar el origen y la existencia de cada color que se encuentra en la naturaleza sería, probablemente, la particular forma, composición o proporción de los elementos básicos que la componen y que se han constituido por ocurrencia de algún proceso natural. Si aceptamos la restricción de que la llama del objeto debe ser un fuego puro, para que sea detectable por su semejanza con la llama visual (Timeo 45b6-d3), debemos pensar que la forma geométrica base es la pirámide (a pesar de que el ojo pueda también añadir icosaedros). Así, pues, el aspecto cromático específico depende en última instancia de las descripciones específicas de esos factores en los que se podrían encontrar, como digo, las especificidades de la forma piramidal (y del icosaedro), así como la combinación o la

\footnotetext{
${ }^{21}$ Según Struycken (2003, p. 301), otra posible explicación es que se refiera a la tierra rojiza de Grecia.
} 
proporción de dichas partículas elementales. Por eso, se puede dudar de que el vocabulario cromático griego se refiera de colores tonales. La semejanza cromática no parece establecerse por la fenomenología del tono, sino por otra serie de criterios no tonales como pueden ser su cercanía material o utilidad, por ejemplo. Parece que no hay más que objetos coloreados, con lo que todo color se asocia con algún determinado conjunto de objetos, ya sea por su semejanza en alguna de las diversas dimensiones (vitalidad, valía, belleza, nobleza, cercanía material, utilidad. . . ) o por algún otro elemento común. La extensión de los conceptos cromáticos se establecería entonces por este tipo de criterios no tonales. ${ }^{22}$ Después, de cada objeto coloreado se podría predicar si es saturado o si es brillante. Y eso Platón lo explica, en el nivel físico, relacionando cada uno de ellos con el tamaño y la velocidad relativa a la que sus respectivas partículas recorren el espacio. Por ejemplo, lo que hace que algo sea rojo sería la particular forma (pirámides e icosaedros), composición y proporción de sus partículas elementales. No obstante, sería el tamaño y la velocidad relativa a la que esas partículas se expanden en el espacio lo que haría que, en su caso, fuese saturado o brillante.

\section{Realismo acerca de los colores}

Hemos favorecido la interpretación de la doctrina de los colores de Platón según la cual los colores que percibimos tienen una naturaleza profunda que si fuera accesible sería identificable a partir de su estudio. Los colores tienen una naturaleza esencial (independiente de los sujetos que las perciben $)^{23} \mathrm{y}$, si ésta fuera detectable, parece que, en principio, se podría identificar. En ese sentido, la doctrina platónica ensaya un primer intento serio, aunque todavía rudimentario, en la dirección de una futura ciencia de los colores. De ahí se sigue, a mi entender, la legitimidad de situar su doctrina en la antesala de las teorías realistas contemporáneas y que, en consecuencia, también ella pueda clasificarse como una doctrina realista. En lo que sigue trataré de defender la legitimidad de dicha conclusión frente a la acusación de que Platón no aceptaría que su doctrina, así descrita, sin ninguna alusión a las Formas cromáticas, se clasificara como realista.

\footnotetext{
${ }^{22}$ Una consecuencia notable de esa divergencia con nuestros conceptos cromáticos es que los conceptos cromáticos griegos no se podrían representar como los nuestros en un espacio tridimensional. Por lo tanto, tampoco permitiría el tipo de naturalización fisicista a la que estamos acostumbrados, por ejemplo, su reducción a algún rango de reflectancia espectral de las superficies.

${ }^{23}$ Aunque metafísicamente dependen de su función teleológica en relación con el acto de percibir.
} 


\subsection{El realismo cromático según Platón ${ }^{24}$}

En el Timeo (67c3-68d7), Platón ofrece un modelo posible de naturalización de los colores donde especula sobre sus correspondientes identidades básicas, así como sobre su origen natural. No obstante, tanto la metodología que utiliza como el modelo de explicación que esboza encajan perfectamente dentro de lo que hoy día consideramos como teorías realistas de los colores. Creo que eso debería ser suficiente para clasificar la doctrina de los colores de Platón como un antecedente de las teorías realistas. Debo aclarar, sin embargo, que con ello no pretendo afirmar que Platón se hubiese concebido como proponente de una doctrina realista de los colores. De hecho, habitualmente se da por sentado que para él la realidad está poblada de Formas y que éstas son el único objeto genuino del conocimiento. Por eso, hay razones para creer que Platón no calificaría de realista su doctrina acerca de los colores sin antes confirmar la existencia de las Formas cromáticas. Y es ciertamente discutible que Platón aceptara su existencia. ${ }^{25} \mathrm{Al}$ menos, la interpretación realista que trato de defender no se compromete necesariamente con la existencia de las Formas cromáticas. Así, si clasifico su doctrina como realista es porque no estoy utilizando el rubro "realista" en el sentido que normalmente se le atribuye; lo utilizo en el sentido contemporáneo, un uso compatible con que no haya Formas cromáticas. Intencionadamente, hago mi interpretación compatible con que Platón negara que su doctrina de los colores sea realista (si negara la existencia de las Formas cromáticas) ya que únicamente afirmo que aplicando los estándares ontológicos y epistémicos actuales a su doctrina de los colores ésta quedaría perfectamente clasificada como realista.

Ignoramos qué pensaba Platón sobre la existencia de las Formas cromáticas. Pero sabemos que era pesimista sobre el conocimiento que se puede lograr en cuanto a la naturaleza de los colores. Afirma, por ejemplo, que ese tipo de conocimiento (de perfil bajo en caso de que no existan las Formas cromáticas) sobre qué son los colores

\footnotetext{
${ }^{24}$ Agradezco a uno de los árbitros de Crítica que me haya señalado la conveniencia de aclarar este punto.

${ }^{25}$ La distinción entre la blancura y el color blanco (Teéteto 156e4-5, 182bl-2) parece sugerir que sí aceptaría su existencia (McDowell 1973, pp. 139-140). Sin embargo, el hecho de que las Formas se autoprediquen y sean incoloras (Fedro 247c6-7) parece sugerir lo contrario. Carecemos de elementos de juicio definitivos para decidir la cuestión de un modo resolutivo. Sin embargo, a mi entender, mi interpretación es compatible tanto con la existencia de las Formas cromáticas, como con su negación, y con varias maneras metafísicas de entenderlas.
} 
y cuál es su función es divino, sólo Dios dispone de él, no el hombre, ya que sólo Dios sabe cómo convertir lo uno en múltiple y lo múltiple en uno (Timeo 68b6-8, 68c7-d7). Esta idea me merece al menos dos comentarios. El primero es que Platón no nos dice cuál es la constitución exacta de los colores. Muestra su necesidad, es decir, el proceso natural que los ha generado (si creemos que las formas geométricas son coloreadas) o el vínculo entre las formas geométricas y las sensaciones cromáticas que éstas generan (si creemos que las formas geométricas no son coloreadas). Pero, en cualquiera de los casos, admite ignorar su naturaleza última. Esto representa, en mi opinión, una prueba más de la interpretación realista, según los estándares actuales, ya que señala precisamente el tipo de conocimiento al que el realista aspiraría actualmente. El segundo comentario es sobre su profecía, epistemológicamente más comprometida que la mera constatación de la ignorancia, de que el hombre nunca llegará a conocer la naturaleza constitutiva de los colores (Timeo 68d6-7). El hecho de que ese tipo de conocimiento esté vedado al ser humano puede significar dos cosas, o bien que por alguna razón los colores sean incognoscibles per se para los humanos, o bien que para aquellos griegos en particular eran inaccesibles de hecho. Yo simpatizo con la segunda interpretación (Txapartegi 2008): creo que Platón aceptaría que si algún día pudiéramos acceder a los colores, podríamos identificar su composición natural; es decir, podríamos identificar su constitución geométrica (si creemos que las formas geométricas son coloreadas) o podríamos conocer el vínculo de necesidad entre las formas geométricas y las sensaciones cromáticas que éstas generan (si creemos que las formas geométricas no son coloreadas). ${ }^{26}$ Entiendo que su vaticinio responde más a la falta de perspectiva de la evolución de las capacidades epistémicas humanas para acceder al nivel de las partículas, que a una supuesta naturaleza incognoscible de las mismas (como asumió la interpretación subjetivista). Creo que cuentan como razones favorables a esta interpretación el lugar del Timeo donde sitúa la discusión, así como la falta de una alusión explícita a aquello diferente en la naturaleza de los colores que los haría incognoscibles per se.

En definitiva, puede ser que para Platón el único saber sobre los colores a los que el filósofo puede aspirar sea de perfil bajo (según sus estándares epistémicos), exhaustivamente compuesto por opiniones

\footnotetext{
${ }^{26}$ Incluso en el caso de que la necesidad involucrada en los procesos se nos escape, creo que podríamos llegar a conocer la dependencia ontológica de los mismos en relación con las formas geométricas.
} 
verosímiles sobre entidades geométricas particulares y sus relaciones causales. Ahora, si para nosotros ese tipo de saber (cuando es verdadero) es suficiente para constituir conocimiento genuino sobre la identidad y la génesis natural de los colores, nosotros deberíamos aceptar el espíritu realista de su propuesta, y clasificarlo como tal. Quizá, si nos situáramos en la perspectiva de sus propios supuestos ontológicos y epistemológicos, una forma más neutral que realista para definir su posición sería "naturalista". Sin embargo, la intención de este artículo no es meramente exegética. Trato de recuperar la doctrina de los colores de Platón para el debate contemporáneo, de manera que los defensores actuales de las teorías realistas (tanto del fisicismo como del primitivismo) puedan legítimamente apelar a ella para que figure como el antecedente de los aspectos centrales de su propuesta. Por eso considero que es importante continuar clasificando su propuesta como realista, desde los parámetros actuales, a pesar de que no podemos decidir si él aceptaría ese rótulo para sí mismo (porque carecemos de los elementos de juicio necesarios para decidir si aceptaba la existencia de Formas cromáticas).

\subsection{Variantes de realismo cromático}

Una clara ventaja de clasificar su doctrina de los colores como realista es que permite aplicar directamente sobre ella algunas de las variantes del realismo cromático contemporáneo. En este sentido general, y si mi interpretación es correcta, Platón podría coincidir al menos con dos posiciones filosóficas contemporáneas, el fisicismo y el primitivismo, ya que para ambas la idea fundamental es que los colores tienen naturalezas esenciales (independientes de los sujetos perceptores) que, en principio, se pueden conocer. ${ }^{27}$ Pero, ¿a cuál de las dos se acerca más la doctrina de los colores de Platón? Considero que examinar su doctrina desde esta perspectiva repercute positivamente en una mejor comprensión de la posición de Platón, a pesar de que, como se verá, los resultados de esta comparación vienen limitados por la escasez de evidencia textual disponible.

Recordemos que la principal tesis del fisicismo es que los colores son propiedades físicas de los objetos físicos (superficies, volúmenes, espacios o fuentes de luz). ${ }^{28}$ Esto es, son propiedades complejas, categóricas y, probablemente, monádicas de los objetos. Normalmente

\footnotetext{
${ }^{27}$ Para una cartografía del fisicismo, véase Txapartegi 2004; del primitivismo, Byrne y Hilbert 2007.

${ }^{28}$ El fisicismo acerca del color es, sin duda alguna, la propuesta realista que actualmente cuenta con mayor apoyo dentro de la filosofía analítica: Lycan 1996,
} 
también se considera que los colores son propiedades constantes (con la posible salvedad de los objetos iridiscentes) y que están relacionados con la luz. En definitiva, la manera más general de ser fisicista es afirmando que los colores son las propiedades físicas - complejas, categóricas y, probablemente, monádicas - de los objetos, constantes y relacionadas con la luz, que normalmente causan que el sujeto perciba que un objeto dado sea de tal o cual color. Con un espíritu muy parecido, el primitivismo cromático dice que los colores son propiedades primitivas, sui generis, no reducibles a propiedades físicas más básicas y no dependientes de la respuesta perceptiva de ningún sujeto. Ontológicamente, la diferencia más notable entre estas dos posiciones es que el primitivismo rechaza la reducción de los colores a propiedades físicas más básicas. Sin embargo, también hay una diferencia epistemológica importante. El primitivismo acepta normalmente la tesis de la suficiencia perceptiva, mientras que el fisicismo, por el contrario, dice que para conocer la naturaleza de los colores es necesario el concurso de la ciencia. Por lo tanto, la percepción común no es suficiente. Lo que trataré de hacer a continuación es utilizar los criterios más importantes sobre los cuales difieren los fisicistas y los primitivistas para determinar a cuál de ellos podría ser más afín la doctrina de los colores de Platón.

\subsection{Fisicismo}

En el Timeo (67c3-68d7), Platón trata de presentar las naturalezas constitutivas de los colores, aunque al final admite que desconoce cuáles sean éstas. Se limita, simplemente, a describir cuál podría ser el proceso natural que las origina. Ese intento es filosóficamente significativo porque el mero hecho de que esas naturalezas constitutivas de los colores existan y se puedan conocer (según nuestros estándares epistémicos) abre la posibilidad de que, en principio, haya dos accesos epistémicos a los colores, uno perceptivo y otro reflexivo, el primero que conduce al color percibido (la sensación cromática) y el segundo que conduce a su naturaleza profunda (las formas geométricas que en la naturaleza constituyen cada color). Además, el hecho de que sólo los dioses conozcan su naturaleza profunda pone en evidencia que la percepción ordinaria no es suficiente para conocerla. La pregunta es: si todo esto es cierto, ¿cuál de los dos constituye la referencia de los conceptos cromáticos, el color percibido o su naturaleza profunda?

Shoemaker 1996, Lewis 1997, Ross 2000, Tye 2000, Byrne y Hilbert 2003, Searle 2004 o Jackson 2007. 
Si Platón identifica cada color con la forma, combinación y proporción de sus partículas fundamentales, sin hacer alusión al aspecto fenoménico, ésa parece ser una reducción del tipo fisicista. En este sentido parece que la mera pretensión de mostrar la génesis de la naturaleza esencial de los colores es suficiente para apoyar su lectura fisicista. Por lo tanto, según esta interpretación fisicista, Platón favorecería el acceso reflexivo al perceptual. En su sentido estricto, los colores no serían propiedades, serían objetos (porque son llamas o partículas), pero categóricamente serían atribuibles a los objetos vistos, al igual que el fuego es atribuible al objeto que se quema. Es decir, los colores serían objetos complejos, aunque monádicamente atribuibles a los objetos que las desprenden. De hecho, cuando Platón distingue la blancura del color blanco (Teéteto 156e4-5, 182b1-2), parece que con la blancura pretende referirse de aquello categórico, monádico, complejo, constante y sin aspecto cualitativo que despiden algunos objetos y que, en circunstancias normales, interactuando con el flujo visual causa que dichos objetos se vean blancos.

En contra de esta lectura fisicista se puede objetar que es precipitado atribuírsela a Platón. Las partículas que se identifican con los colores no se perciben como partículas. No sabemos cuál sea su figura, composición y proporción porque no podemos verlas como partículas. Según la lectura fisicista, esto se debería a que son demasiado pequeñas (y no a una naturaleza incognoscible per se). Por eso, podemos preguntarnos si, a su vez, esas partículas tan pequeñas que constituyen los flujos que son los colores están coloreadas. La lectura fisicista estándar de Platón estaría comprometida con una respuesta negativa a esa pregunta. Al igual que para que la madera sea vista como blanca se necesita, además de la blancura, el concurso del flujo visual, para que esas partículas sean vistas como blancas quizá también se necesite la participación activa del flujo visual. No obstante, eso no es posible porque en el nivel de las partículas no hay flujo visual. De hecho, como dice McDowell, en el Teéteto nada indica que los colores (los flujos objeto) deban estar coloreados (McDowell 1973, p. 183). Las emanaciones son los colores, pero eso no implica que en una descripción más básica de ellas figure alguna fenomenología particular.

Efectivamente, ésa podría ser una interpretación válida. El problema es que no se puede decidir si es correcto atribuírsela a Platón. No podemos decidir si Platón se adheriría a la lectura fisicista. Lo que podemos saber, sin embargo, es que se encontraría con serios problemas si lo hiciera; es decir, el programa fisicista vincula el color real con el color meramente percibido de un modo contingente y eso genera 
algunos conocidos problemas que podrían afectar al Platón fisicista. Si se identifica alguna emanación como blanca, todas las demás emanaciones no podrían serlo o serían blancas sólo ilusoriamente, a pesar de que generen la misma sensación cromática en los seres humanos o en otras especies (excepto en la versión dependiente de la respuesta que veremos más adelante). $\mathrm{Y}$ ese problema se podría generalizar. Si Platón hubiera pretendido reducir el vocabulario cromático a otro no cromático donde cada color puede ser exhaustivamente descrito utilizando únicamente vocabulario geométrico, entonces ocurriría que no sería esencial a la naturaleza de los colores aparecer de tal o cual manera a los sujetos humanos en circunstancias normales. Así, la segunda consecuencia difícil de aceptar es que sería posible que

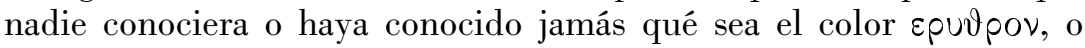
cualquier otro, en realidad. Por lo tanto, como ya hemos dicho, tampoco podría estar seguro de si la extensión que habitualmente asocia a los "objetos rojos" es verdadera, dado que puede haber objetos que se ven rojos y que en realidad no lo son y al revés; es decir, podría ser el caso de pares de objetos metamer (Hilbert 1987), con la misma apariencia pero sin la misma naturaleza. En consecuencia, para saber cuál es el verdadero color de cada objeto, no sería suficiente mirar los objetos envueltos en la luz del día, sino que se debería esperar a su naturalización definitiva. Los expertos (en este caso, los dioses) sabrían mejor que nadie qué es el color rojo y de cuáles objetos se puede predicar verídicamente. ${ }^{29} \mathrm{Y}$, evidentemente, eso viola una creencia básica asociada a la semántica ordinaria de los conceptos cromáticos. Por lo general, creemos que mirar un objeto en circunstancias normales es suficiente para saber cuál es su color. Si creemos que Platón no hubiera querido prescindir de esa creencia básica, debemos concluir que no aceptaría una relación meramente contingente entre el color real y el color percibido.

La situación filosófica en la que se encontraría se puede reformular de la siguiente manera. Una vez que se identifica la blancura con una emanación particular, se abre la posibilidad de una lectura fisicista y, en consecuencia, de una pregunta incómoda: ¿es blanca la blancura (la emanación)? No sabemos qué respondería Platón, pero, ¿no resulta extraño que no lo podamos saber? ¿No resulta extraño que sea posible que la blancura no sea blanca? Da la impresión de que, en

\footnotetext{
${ }^{29}$ Es verdad que Platón dice que los dioses conocen la naturaleza profunda de los colores, y que saben cómo generar los colores en la naturaleza, pero de ahí no concluye que los dioses pueden clasificar cromáticamente el entorno mejor que cualquier sujeto humano con visión normal.
} 
manos de Platón, conceptos como "blancura" cambian su significado y dejan de significar propiedades que son esencialmente cualitativas. Y no sabemos si ésa era su intención. La crítica es que si asociamos Platón al fisicismo, y la identidad de los colores no se vincula esencialmente con sus correspondientes apariencias perceptuales, entonces los conceptos cromáticos dejan de concebirse como conceptos experienciales. ¿Estaría Platón dispuesto a aceptar esa consecuencia? Puede ser que sí. En la filosofía contemporánea es posible encontrar varios ejemplos. Searle (2004, pp. 12ls), por ejemplo, afirma que los colores se asocian a su aspecto sólo contingentemente. Averill asegura que los colores no tienen que ser esencialmente cualitativos ${ }^{30}$ y Smart propone el disyuntivismo para evitar, de una manera ad hoc, la consecuencia fisicista de que hasta pasar por el laboratorio no podemos saber si dos objetos tienen el mismo color (Smart 1975). No es descabellado suponer que Platón también podría adoptar cualquiera de esas posiciones filosóficas.

No obstante, si no quisiera aceptar esas consecuencias, todavía dispondría de la alternativa primitivista. Antes de entrar a explorar esa hipótesis, sin embargo, conviene analizar brevemente la posibilidad de atribuir a Platón otros tipos de fisicismo cromático como el representacionismo ${ }^{31}$ o el dependiente de la respuesta ${ }^{32}$ que no postulan una relación contingente entre el color real no cualitativo y el color percibido esencialmente cualitativo.

\subsection{Variantes fisicistas}

Creo que es bastante fácil mostrar por qué Platón no subscribiría el fisicismo representacionista. Según la tesis específica del fisicismo representacionista, el color percibido se puede reducir exhaustivamente a la propiedad física básica que se identifica con el color. La relación entre el color percibido y el color físico no es contingente, como originalmente propone el fisicismo, sino necesaria, ya que entre ambas se postula una relación de identidad. La blancura (la propiedad física) determina que la veamos blanca (el aspecto cualitativo), porque el color blanco que vemos (el aspecto cualitativo) no es más que un acceso representacional o funcional a la blancura (la propiedad física

\footnotetext{
${ }^{30} \mathrm{Y}$, por lo tanto, los conceptos esencialmente cualitativos no se refieren de los colores: "Yellow is not a color" (Averill 1985, p. 301).

${ }^{31}$ Dos formulaciones recientes de la posición representacionista son Tye 2000 y Jackson 2007.

${ }^{32}$ Algunas formulaciones recientes del fisicismo dependiente de la respuesta son Wright 1998, Ross 2000 y Jackson y Pettit 2002.
} 
particular que se encuentra en los objetos que se ven blancos). Esta propuesta coincide con la platónica en que, por ejemplo, para definir la blancura (el color) no parece necesario apelar al color blanco (el color percibido). La blancura se presenta precisamente como una propiedad física específica que, incidentalmente, causa que algo se vea (se represente) como blanco. La causa es incidental porque supone un sistema perceptual humano cuya función es detectar y distinguir precisamente esa propiedad física. Así, la identidad de la blancura se define independientemente de su función procreadora del color blanco (cuando interactúa causalmente con el flujo visual), y puede ser descrita tomando en cuenta únicamente la forma, la combinación y la proporción de sus partículas básicas. La coincidencia de las dos posiciones parece notable. Sin embargo, ésta no podría ser la propuesta de Platón. Según Platón, el color blanco existe como un ente ontológicamente (aunque no causalmente) independiente de la blancura. En ese sentido, el color blanco no es reducible a la blancura y, por lo tanto, no puede haber una relación de identidad entre ambas como postula el representacionismo fisicista. Tomando en cuenta la distinción que traza en el Teéteto entre el color blanco y la blancura, es natural suponer que se refieren de entidades ontológicamente distinguibles, con lo que no se cumpliría la premisa básica del representacionalismo que postula una relación de identidad entre la propiedad física representada y el aspecto fenoménico asociado a aquélla.

Respecto del fisicismo dependiente de la respuesta es más difícil determinar cuál hubiera sido la posición de Platón. Por un lado, parece que cada flujo particular que se identifica con un color puede ser caracterizado independientemente de cualquier respuesta perceptual que pueda llegar a generar. Esto refuerza la idea de que Platón no aceptaría ningún tipo de dependencia de los flujos respecto de la sensación cromática que generan. Sin embargo, la función teleológica que Platón introduce como una parte esencial de la identidad de los colores parece reforzar la intuición contraria. Cada color adquiere parte de su identidad porque tiene la función de generar tal o cual sensación cromática en los sujetos humanos normales en circunstancias normales (para que éstos puedan ver los objetos a distancia). Por lo tanto, parece que la identidad de cada color depende ontológicamente, a través de su función, de la respuesta cromática que genera. Eso no implica que los flujos que son los colores dependan de los sujetos para existir. Los flujos podrían existir perfectamente en un mundo sin sujetos. Además, si ese mundo coincidiese con el nuestro en los flujos que despiden los objetos, podríamos dar por hecho 
también que ese mundo coincidiría con el nuestro en sus colores. $\mathrm{Y}$, no obstante, aceptar dicha dependencia funcional de la respuesta generaría un problema.

Como acabamos de decir, los flujos no tienen por qué ser dependientes de la respuesta. Sin embargo, los colores serían, según esta hipótesis, funcionalmente dependientes de la respuesta. En consecuencia, y dada la identidad entre las emanaciones y los colores (reales, no los percibidos), cada lado del predicado de identidad contendría propiedades diferentes. El color real sería funcionalmente dependiente de la respuesta, y la emanación no. Frente a esta situación, y dado que no puede haber una relación de identidad entre dos entidades que tienen propiedades diferentes, Platón debería rechazar la identidad entre el color real y la emanación. O al menos debería matizarla, en el siguiente sentido. Si los colores son dependientes de la respuesta de una manera esencial, es decir, si su ser color depende de generar cierta respuesta cromática en sujetos humanos normales, el día en que los dioses cambiasen la función de los flujos, y éstos no tuviesen ya más la función de generar ninguna respuesta cromática en sujetos humanos normales, ese día esos mismos flujos dejarían de ser colores. Imaginemos, por ejemplo, que un flujo particular es "amarillo" si tiene como función generar la respuesta cromática adecuada en los sujetos humanos normales en circunstancias normales. Cuando dejase de tener esa función, ese mismo flujo dejaría de ser amarillo. Pero mientras los flujos continúen teniendo esa función, continuará siendo verdadera la identidad entre el color real y la emanación. Desgraciadamente, tal como ocurre en el caso del fisicismo estándar, no es posible saber si Platón aceptaría esa conclusión.

Muchos filósofos contemporáneos han tratado de solventar los problemas que les genera el fisicismo estándar avanzando sobre la vía del fisicismo dependiente de la respuesta. Sin embargo, y dada la evidencia textual disponible, no creo que sea posible determinar si Platón habría seguido ese camino.

\subsection{Primitivismo}

Quienes no simpaticen con la lectura fisicista de Platón, porque no creen que Platón haya alterado la semántica de los conceptos cromáticos o no creen que haya reducido el color percibido al color físico, todavía pueden recurrir a la lectura primitivista. La tesis distintiva del primitivismo es que la naturaleza de los colores, aunque independiente de los sujetos que las perciben, involucra esencialmente su aspecto fenoménico. Según la lectura primitivista, Platón podría 
pensar, por ejemplo, que las esencias cromáticas describen su naturaleza profunda y que éstas son, a su vez, esencialmente fenoménicas, i.e., contienen esencialmente algún carácter cualitativo. Es decir, la blancura (la emanación) sería ella misma blanca (incluso fuera del acto perceptual). Esta posición evitaría algunos problemas asociados a la interpretación fisicista o representacionista para la cual en la descripción fundamental de los objetos no figura el aspecto fenoménico. No habría que alterar la semántica habitual asociada a los conceptos cromáticos ni habría que reducir el color percibido al color físico. Ocasionaría, sin embargo, otra serie de rompecabezas.

El particular, el primitivismo no puede escapar completamente de las acusaciones anteriores si sigue postulando una relación contingente entre la esencia cromática y su apariencia en los sujetos humanos normales en circunstancias normales. Esa relación sería contingente, por ejemplo, si pudiera ser el caso de que los objetos tengan una apariencia cromática dada, pero que los humanos sean incapaces de percibirla o que la perciban de otra manera. Ahora bien, si la relación es meramente contingente y no tenemos otras vías epistémicas para conocer el aspecto de los colores que la vista, ¿cómo sabemos que percibimos el color real de los objetos? Según esta hipótesis, la percepción normal no garantizaría la veracidad de nuestras atribuciones cromáticas. Y eso implicaría, de nuevo, otra semántica asociada a esos conceptos. La única garantía disponible para Platón estaría sujeta a la fe de que los dioses no nos habrían provisto de ojos para alcanzar a ver propiedades que no son así en realidad; es decir, debería refugiarse en la garantía teológica de que la relación entre el color real y el percibido no es contingente, sino divinamente necesaria.

Pero, en ese caso, si Platón se empeñara en postular una relación de necesidad entre los colores y nuestro acceso perceptual a ellos, surgiría otro problema también importante: el chovinismo de especie. Esto es, si pensase que nuestra percepción cromática es necesariamente verdadera pero intuyese que otras especies animales poseen percepciones cromáticas del entorno que son divergentes de la nuestras, como parece razonable intuir, entonces debería concluir que las demás especies no perciben correctamente la composición cromática del entorno. Eso supondría que cualquier sistema visual cromático divergente del humano engaña sistemáticamente a quienes gozan de él y, normalmente, ningún filósofo desea comprometerse con una afirmación empírica de semejante calado. ${ }^{33}$ ¿Estaría dispuesto Platón a

\footnotetext{
${ }^{33}$ Cabría una posible salida si Platón estuviese dispuesto a asumir que cada especie detecta diferentes combinaciones de partículas elementales. Dado que Platón mani-
} 
adoptar para sí esas dos posiciones filosóficas, la garantía de la religión y el chovinismo de especie? Tal vez sí, pero carecemos de elementos de juicio para poder afirmarlo. Ahora bien, si no lo hiciera, el primitivismo lo conduciría a la misma incómoda situación epistémica que el fisicismo, y lo forzaría a violar creencias básicas asociadas a los conceptos cromáticos, como que no podríamos saber si el oro es en verdad amarillo y brillante, a pesar de que normalmente lo veamos así.

En resumen, comparando a Platón con las posiciones contemporáneas, creo que, ontológicamente, no disponemos de evidencia textual suficiente para decidir si simpatizaría con el fisicismo o con el primitivismo, porque para ello sería crucial saber si la blancura, por ejemplo, es esencialmente blanca. Dada la distinción platónica entre la blancura y el color blanco, parece que no lo sería, pero no podemos determinar si estaría dispuesto a aceptar sus consecuencias o si preferiría explorar otras rutas filosóficas como el primitivismo. Epistemológicamente, en cambio, pienso que estaría más cerca del fisicismo porque, según Platón, para identificar la naturaleza de un color no basta con percibirlo. Se requiere un tipo más profundo de investigación y esa habilidad, según reconoce, en aquella época era una posesión exclusiva de los dioses.

\section{Conclusión}

En la primera sección he tratado de describir la doctrina de Platón acerca de los colores. Parece ser que, según Platón, la identidad de cada color viene determinada por la particular combinación de las pirámides constitutivas de los fuegos que despiden los objetos y que, a su vez, ha sido generada como resultado de un proceso natural. Además, esa naturaleza profunda de los colores sería perfectamente cognoscible (según los estándares epistémicos actuales), aunque sea inaccesible al ojo humano. También he tratado de argumentar que, dada su ignorancia acerca de las identidades particulares asociadas a cada color, Platón se limita a mostrarnos cuál podría ser el proceso natural que las genera, así como a diseñar un modelo explicativo de cómo podría ser que algunos de ellos son saturados y brillantes. Por desgracia, no se ha reconocido suficientemente el esfuerzo

fiesta abiertamente su desconocimiento sobre las bases naturales que se identificarán con cada color, pudiera ser el caso de que lo que despidan los objetos coloreados sea tan complejo que cada especie animal detecte únicamente aquello que le interesa, siendo cada uno de ellos un posible color. Por lo tanto, los objetos no tendrían un único color real, sino un rango posible de colores, de los cuales cada sistema perceptivo escoge el que más le conviene. 
magistral de Platón al distinguir dos tipos de explicación: por un lado, el de los colores particulares y, por otro, el de las cualidades asociadas a los colores como su saturación y brillo. En la segunda sección he argumentado que, según los estándares epistémicos y ontológicos actuales, la doctrina de los colores de Platón debería ser clasificada como realista. Sin embargo, he presentado las dificultades que encontraríamos al clasificar su propuesta bajo cualquiera de las marcas realistas actuales. Ontológicamente, no podríamos decidir si asociarla al fisicismo o al primitivismo, aunque, epistemológicamente, se asociaría más fielmente al fisicismo.

En definitiva, y si mi interpretación realista es correcta, hay mucho que hoy por hoy sigue siendo rescatable de la doctrina de los colores de Platón. En particular, considero que el Timeo (67c3-68d7) ilustra un intento pionero en la búsqueda de la identidad profunda de los colores y que es precisamente ahí donde reside su principal valor: ser uno de los ancestros de la ciencia moderna de los colores, con lo que se legitima su introducción al debate contemporáneo como el precursor de las teorías realistas del color. ${ }^{34}$

\section{BIBLIOGRAFÍA}

Averill, E.W., 1985, "Color and the Anthropocentric Problem", Journal of Philosophy, vol. 82, pp. 281-304.

[Pseudo-Aristóteles] De Coloribus, trad. al inglés T. Loveday y E.S. Forster, Oxford University Press, Oxford.

Aristóteles, Del Sentido y lo sensible, trad. F. Samaranch, Aguilar, Madrid. Burnyeat, M.F., 1999, "Knowledge is Perception", en G. Fine (comp.), Plato: Metaphysics and Epistemology, Oxford University Press, Oxford, pp. 320-354.

— 1990, The Theaetetus of Plato, Hackett, Nueva York.

Byrne, A. y D. Hilbert, 2007, "Color Primitivism", Erkenntnis, vol. 66, pp. 73-105.

— 2003, "Color Realism and Color Science", Behavioral and Brain Sciences, vol. 26, pp. 52-63.

Cornford, F.M., 1937, Plato's Cosmology, Bobbs-Merrill, Nueva York.

${ }^{34}$ Quiero agradecer a María Angélica Fierro, Ricardo Salles, Rocío Cázares, Andoni Ibarra y, muy especialmente, a Andrea Lozano sus comentarios a versiones anteriores del presente trabajo. También quiero agradecer al Instituto de Investigaciones Filosóficas, así como a la Universidad Autónoma de Zacatecas y la Universidad Autónoma del Estado de Morelos, en Cuernavaca, por invitarme a presentar partes del trabajo. Por último, agradezco también a los árbitros anónimos de Crítica por sus comentarios y sugerencias, los cuales han permitido reforzar considerablemente el presente artículo. 
Gage, J., 1999, Colour and Meaning, Thames and Hudson, Londres.

—_, 1993, Colour and Culture, Thames and Hudson, Londres. [Versión en castellano: Color y cultura, trad. Adolfo Gómez Cedillo y Rafael Jackson Martin, Siruela, Madrid, 1993.]

Gulley, N., 1962, Plato's Theory of Knowledge, Methuen, Londres.

Hilbert, D., 1987, Color and Color Perception: a Study in Anthropocentric Realism, CSLI, Stanford.

Ibarra, A. y E. Txapartegi, 2005, "Fisicalismo científicamente compatible", Revista de Filosofía, vol. 30, pp. 39-59.

Ierodiakonou, K., 2005, "Plato's Theory of Colours in the Timaeus", Rhizai: A Journal of Ancient Philosophy and Science, vol. 2, no. 2, pp. 219-233.

— inédito, "Basic and Mixed Colours in Empedocles and in Plato".

Jackson, F., 2007, "Colour for Representationalists", Erkenntnis, vol. 66, pp. 169-185.

Jackson, F. y P. Pettit, 2002, "Response-Dependence Without Tears", Philosophical Issues, vol. 12, pp. 97-117.

Lewis, D., 1997, "Naming the Colours", Australasian Journal of Philosophy, vol. 75, pp. 325-342.

Lycan, W., 1996, Consciousness and Experience, MIT Press, Cambridge, Mass.

McDowell, J., 1973, Plato: Theaetetus, Oxford University Press, Oxford.

Platón, 1992, Timeo, trad. F. Lisi, Gredos, Madrid. También en Platón: Obras Completas, trad. F. Samaranch, Aguilar, Madrid, 1966.

— 1988, Teéteto, trad. A. Vallejo Campos, Gredos, Madrid.

—, 1983, Menón, trad. F.J. Olivieri, Gredos, Madrid.

Ross, P., 2000, "The Relativity of Color", Synthese, vol. 123, pp. 105-129.

Searle, J., 2004, Mind, Oxford University Press, Oxford.

Shoemaker, S., 1996, "Colors, Subjective Relations and Qualia", en E. Villanueva (comp.), Philosophical Issues 7. Perception, Ridgeview, Atascadero, pp. 55-66.

Smart, J.J.C., 1975 (1997), “On Some Criticisms of a Physicalist Theory of Colors", en Byrne y Hilbert (comps.), Readings on Color, MIT Press, Mass., Cambridge, pp. 1-11.

Struycken, P., 2003, "Colour Mixtures According to Democritus and Plato", Mnemosyne, vol. 61, pp. 273-305.

Taylor, A.E., 1928, A Commentary on Plato's Timaeus, Oxford University Press, Oxford.

Taylor, C.C.W. (comp.), 1999, The Atomists: Leucippus and Democritus, University of Toronto Press, Toronto.

Teofrasto, Sobre las sensaciones, trad. J. Solana Dueso, Anthropos, Barcelona.

Txapartegi, E., 2008, "Platón sobre los colores", Teorema, vol. 27, no. 2, pp. $5-25$.

— 2004, Color y representación, Universidad del País Vasco, Bilbao. 
Tye, M., 2000, Consciousness, Color, and Content, MIT Press, Cambridge, Mass.

Van Brakel, J. y B. Saunders, 2002, "The Trajectory of Colour", Perspectives on Science, vol. 10, no. 3, pp. 302-355.

Wright, C., 1998, "Euthyphronism and the Phisicality of Colour", European Review of Philosophy, vol. 3, pp. 12-48.

Recibido el 7 agosto de 2007; revisado el 1 de febrero de 2008; aceptado el 3 marzo de 2008. 